\title{
SIMPLE KNOTS WITHOUT UNIQUE MINIMAL SURFACES
}

\author{
HERBERT C. LYON
}

\begin{abstract}
Abstracr. We construct infinitely many simple knots and infinitely many nonsimple knots, all of genus one, and all having both knotted and unknotted minimal spanning surfaces. The complements of the simple knots all contain closed incompressible surfaces of genus two.
\end{abstract}

I. Introduction. A knot is simple if every incompressible torus in its complement is boundary parallel; i.e., if it has no companions (cf. Schubert [8]). A surface in $S^{3}$ is unknotted if the fundamental group of its complement is free; otherwise the surface is knotted. In this paper we establish the existence of an infinite class of simple knots of genus one, each of which has both knotted and unknotted minimal spanning surfaces, thus providing an affirmative answer to a question posed in [2]. The complement of each of these knots contains a closed incompressible surface of genus two. We also establish the existence of an infinite class of nonsimple genus one knots, each of which has both knotted and unknotted minimal spanning surfaces. Although Alford, Daigle, and Schaufele ([1], [2], [3], [4]) have constructed knots with nonunique minimal spanning surfaces, none of theirs is simple. All work is done in the PL category, all knots are tame in $S^{3}$, all surfaces are orientable, and the notation and terminology follow [6] and [7].

II. The construction. Let $T$ be a torus which determines the genus one Heegaard splitting $(U, V)$ of $S^{3}$, and let $u$ and $v$ be nonseparating disks properly embedded in $U$ and $V$, respectively, such that $u \cap v$ is one point. Let $A^{\prime} \subset T$ denote an annular regular neighborhood, in $T$, of the torus knot $(3,4)$ drawn on $T$, and let $A=\mathrm{Cl}\left(T-A^{\prime}\right)$. We may assume that $A$ and $A^{\prime}$ each meet $\operatorname{Bd}(u)$ in exactly three arcs and $\operatorname{Bd}(v)$ in exactly four arcs. Let $\alpha \subset v$ be a properly embedded arc connecting the two components of $\operatorname{Bd}\left(A^{\prime}\right)$ and meeting separate components of $A^{\prime} \cap \operatorname{Bd}(v)$ which are nonadjacent in $\operatorname{Bd}(v)$.

Received by the editors November 29, 1972 and, in revised form, March 19, 1973.

AMS (MOS) subject classifications (1970). Primary 55A25; Secondary 55A05, 55A35.

Key words and phrases. Incompressible surface, knot, knot space, knotted minimal surface, nonunique minimal surface, simple knot, unknotted minimal surface.

(c) American Mathematical Society 1974 
Let $B \subset V$ be a band whose ends lie in distinct components of $\operatorname{Bd}\left(A^{\prime}\right)$, whose median is $\alpha$, and which would make $n$ full twists if the components of $\operatorname{Bd}\left(A^{\prime}\right)$ were moved by an isotopy, in $T$, until they were perpendicular to $\operatorname{Bd}(v)$. Thus $S^{\prime}=A^{\prime} \cup B$ is an orientable surface of genus one with one boundary component, which we choose as our knot $k$; i.e., $k=\mathrm{Bd}\left(S^{\prime}\right)$. Note that $k$ is also spanned by the genus one orientable surface $S=A \cup B$. Let $K$ be the knot space of $k$; i.e., $K=\mathrm{Cl}\left(S^{3}-N(k)\right)$. Since the Alexander polynomial of $k$ is $\Delta(t)=(12 n-6) t^{2}+(13-24 n) t+(12 n-6)$, it follows that $k$ is nontrivial. Hence the genus one spanning surfaces $S \cap K$ and $S^{\prime} \cap K$ must be incompressible. Our knot $k$, for the case $n=1$, is illustrated in the figure.

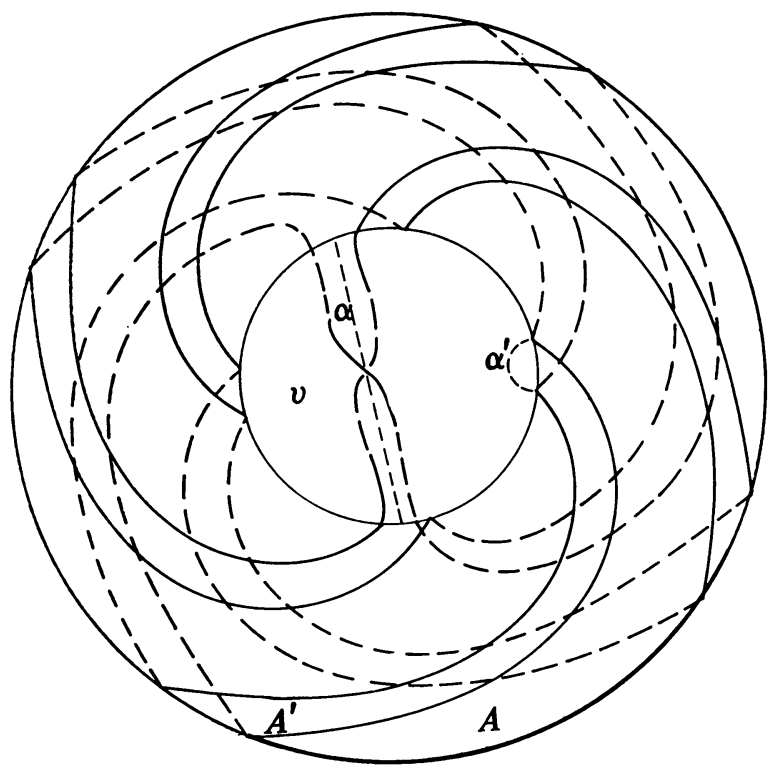

FIGURE 1.

Let $G$ be a group containing an element $g$ of infinite order, and let $a$ be a generator of the infinite cyclic group $Z$. If $m>1$ is a fixed integer and $H$ is the free product of $G$ and $Z$ amalgamated along the subgroups $\langle g\rangle$ and $\left\langle a^{m}\right\rangle$, then we say that $H$ is obtained from $G$ by adjoining an $m$ th root (to $G$ ) along $g$.

THEOREM 1. The surface $S$ is unknotted, while $S^{\prime}$ is knotted.

Proof. Since $B$ may be retracted to $\alpha$, it suffices to consider $A \cup \alpha$ and $A^{\prime} \cup \alpha$. By Van Kampen's theorem, $\pi_{1}\left(S^{3}-(A \cup \alpha)\right)$ is just $\pi_{1}(V-\alpha)$, 
which is free of rank 2, with a cube root adjoined along a generator of $\pi_{1}\left(A^{\prime}\right)$. However, since $A^{\prime}$ meets one component of $v-\alpha$ exactly once, this cube root is adjoined along a primitive element. Hence $\pi_{1}\left(S^{3}-(A \cup \alpha)\right)$ is free and $S$ is unknotted. On the other hand, since every incompressible annulus in a cube-with-handles is boundary compressible, it follows that if $S^{\prime}$ were unknotted, then $A$ would be boundary compressible in $S^{3}-\left(A^{\prime} \cup \alpha\right)$. This would imply that a generator of $\pi_{1}(A)$ is primitive in $\pi_{1}(V-\alpha)$. However, if we let the two components of $v-\alpha$ correspond to generators of $\pi_{1}(V-\alpha)$, we see that a generator of $\pi_{1}(A)$ may have the form $a^{2} b^{2}$, which is not primitive. Hence $S^{\prime}$ is knotted.

Our next goal is to show that $k$ is simple. If not, there exists an incompressible torus $W \subset \operatorname{Int}(K)$ which is not boundary parallel. Let $W$ bound the knot space $L$, put $W$ in general position with $S$, and assume the number of components of $S \cap W$ is minimal. If $S \cap W=\varnothing$, then $L \subset \mathrm{Cl}(K-S)$, contradicting the unknottedness of $S$, and one can easily show that no component of $S \cap W$ is contractible in $W$. Thus $S \cap W$ consists of parallel simple closed curves in $W$ which separate $W$ into annuli $C_{i}, 1 \leqq i \leqq m$. Note that we may assume each $C_{i}$ is properly embedded in $\mathrm{Cl}(K-S)$.

\section{Lemma 1. No component of $S \cap W$ is boundary parallel in $S$.}

Proof. If we assume the contrary, there exists an annulus $E \subset S$ such that one component of $\operatorname{Bd}(E)$ is $\operatorname{Bd}(S)=k$ and the other is $h=E \cap W$. Thus $k$ and $h$ are of the same knot type. But $h$, being drawn on the boundary $W$ of $L$, must be a cable knot about $L$. Hence $\Delta(t)=\rho\left(t^{\gamma}\right) \cdot \sigma(t)$, where $\rho(t)$ is the Alexander polynomial of the knot space $L, \sigma(t)$ is the Alexander polynomial of a torus knot, and $\gamma$ is the absolute value of the intersection number of $h$ with a meridian of $L[5$, p. 144]. Now $\rho(t)$ has even degree and $\Delta(t)$ has degree 2, so if $\gamma>1$ we have $\rho\left(t^{\gamma}\right)=\rho(t)=1$, a contradiction because $\Delta(t)$ is not a torus knot polynomial. If $\gamma=1, W$ is boundary parallel, another contradiction, and the lemma is proved.

Lemma 2. We can move $W$ by an isotopy in $K$ until $W \cap \operatorname{Bd}\left(A^{\prime}\right)=\varnothing$.

Proof. No component of $S \cap W=\bigcup_{i=1}^{m} \operatorname{Bd}\left(C_{i}\right)$ can be contractible in $S$ without contradicting the incompressibility of $W$, so by Lemma 1 and the fact that $S$ is of genus one, all components of $\bigcup_{i=1}^{m} \operatorname{Bd}\left(C_{i}\right)$ are nonseparating, parallel simple closed curves on $S$. Let $A^{\prime \prime} \subset U$ be a properly embedded annulus, parallel to $A^{\prime}$, with $\operatorname{Bd}\left(A^{\prime \prime}\right) \subset A \subset S$. Note that neither component of $\operatorname{Bd}\left(A^{\prime \prime}\right)$ separates $S$ and that $A^{\prime \prime}$ is not parallel to subset of $S$, so if $A^{\prime \prime}$ were boundary compressible with respect to $S$, then $S$ would be compressible, a contradiction. Hence $A^{\prime \prime}$ is boundary incompressible with respect to $S$. Move $A^{\prime \prime}$ an isotopy in $\mathrm{Cl}(K-S)$, keeping $\operatorname{Bd}\left(A^{\prime \prime}\right) \subset S$, 
until $A^{\prime \prime}$ is in general position with $\bigcup_{i=1}^{m} C_{i}$, the intersection $A^{\prime \prime} \cap\left(\bigcup_{i=1}^{m} C_{i}\right)$ contains no contractible simple closed curves, and $\mathrm{Bd}\left(A^{\prime \prime}\right) \cap\left(\bigcup_{i=1}^{m} \mathrm{Bd}\left(C_{i}\right)\right)$ is minimal. This, together with the fact that $S$ is of genus one, assures us that a component of $\mathrm{Bd}\left(C_{i}\right)$ must successively meet alternate components of $\mathrm{Bd}\left(A^{\prime \prime}\right)$ and vice versa. It follows that if $A^{\prime \prime} \cap C_{i}$ were to contain a boundary parallel arc in $C_{i}$, then $A^{\prime \prime}$ would be boundary compressible with respect to $S$, a contradiction, while if $A^{\prime \prime} \cap C_{i}$ were to contain a boundary parallel arc in $A^{\prime \prime}$, then $C_{i}$ would be parallel to a subset of $S$, contradicting the minimality of $S \cap W$. Hence, if $\operatorname{Bd}\left(A^{\prime \prime}\right) \cap\left(\bigcup_{i=1}^{m} \operatorname{Bd}\left(C_{i}\right)\right) \neq$ $\varnothing$, we may assume $A^{\prime \prime} \cap C_{i}$ consists entirely of nonseparating arcs. Now $A^{\prime \prime}$ separates $\mathrm{Cl}(K-S)$, one component of this separation, $U^{\prime}$, is a solid torus, and $A^{\prime \prime}$ has winding number 3 in $U^{\prime}$. But each component of $C_{i} \cap U^{\prime}$ must be a properly embedded disk in $U^{\prime}$ which meets $A^{\prime \prime}$ in exactly two arcs and around which the components of $\operatorname{Bd}\left(A^{\prime \prime}\right)$ alternate. This means that each component $C_{i} \cap U^{\prime}$ is a meridian disk in $U^{\prime}$, contradicting the winding number of $A^{\prime \prime}$. Hence $\operatorname{Bd}\left(A^{\prime \prime}\right) \cap\left(\bigcup_{i=1}^{m} \operatorname{Bd}\left(C_{i}\right)\right)=\varnothing$, and each component of $\operatorname{Bd}\left(C_{i}\right)$ is isotopic, in $S$, to a component of $\operatorname{Bd}\left(A^{\prime \prime}\right)$. But the latter is isotopic, in $S$, to a curve which misses $A^{\prime}$; viz, a component of $\operatorname{Bd}\left(A^{\prime \prime}\right)$ in its original position. It follows that we can move $W$ by an isotopy in $K$ until $W \cap \mathrm{Bd}\left(A^{\prime}\right)=\varnothing$.

As a consequence of Lemma $2, W \cap \operatorname{Bd}(B)=\varnothing$, so we can move $W$ by an isotopy until $W \cap B=\varnothing$. Thus $W$ is incompressible in $S^{3}-$ $(\alpha \cup \operatorname{Bd}(A))$. Put $W$ in general position with $A \cup A^{\prime}$ and assume a minimal intersection, so that if $W \cap\left(A \cup A^{\prime}\right) \neq \varnothing$, then any component $G$ of $W \cap V$ is an incompressible and boundary parallel annulus in $V$ with $\operatorname{Bd}(G)$ parallel to $\operatorname{Bd}(A)$. We may assume that $G \cap v$ consists of four arcs, each disjoint from $\alpha$. But this can happen only if $G$ is parallel to a subset of $A$ or $A^{\prime}$, in which case we can reduce $W \cap\left(A \cup A^{\prime}\right)$, a contradiction. This means that either $W \subset U$ or $W \subset V-\alpha$, both contradictions, since $U$ and $V-\alpha$ are cubes-with-handles. Hence we have proved

\section{THEOREM 2. The knot $k$ is simple.}

III. Other observations. If we construct another knot $k^{\prime}$ as above, using the arc $\alpha^{\prime}$ instead of $\alpha$ and requiring $n \neq 0$, the surface corresponding to $S$ will be unknotted for similar reasons.

Note, however, that $\alpha^{\prime}$ may be assumed to lie in a regular neighborhood of the annulus $A^{\prime}$. Hence the boundary of this regular neighborhood is an incompressible torus bounding a $(3,4)$ torus knot space and disjoint from the surface corresponding to $S^{\prime}$, so the latter surface is knotted and $k^{\prime}$ is not simple. In fact, if $n= \pm 1, k^{\prime}$ is a twisted double of the torus knot $(3,4)$.

In [6, pp. 41-42], we asked if a simple knot space could contain a 
closed incompressible surface of genus greater than one. Our knot $k$ provides an affirmative answer as a consequence of

THEOREM 3. The complement of a simple knot with a knotted, incompressible, genus one spanning surface $S$ contains a closed incompressible surface of genus two.

PROoF. Let the inside of a closed surface in a knot space be the closure of that component of its complement which does not contain the boundary of the knot space, and let the outside be the closure of the other component. We show that the genus two surface $M=\operatorname{Bd}(N(S))$ is incompressible. If $M$ were compressible to the outside, there would exist a disk $D$ in the outside of $M$ with $D \cap M=\operatorname{Bd}(D)$ and $D \cap \operatorname{Bd}(S)=\varnothing$. After putting $D$ in general position with $S$, the incompressibility of $S$ would assure us that we could remove each component of $S \cap D$ by an isotopy of $D$, and $D$ would be parallel to a disk in $M$. Thus we may assume that $M$ is compressible to the inside; i.e., $D$ is a disk inside $M, D \cap M=\operatorname{Bd}(D)$, and $D$ is not parallel to a disk in $M$. If $D$ separates $M$, the components must be tori with disjoint interiors, so at least one is compressible to the inside, and we may replace $D$ by a nonseparating disk, which we again call $D$. Replace the annulus $A=N(D) \cap M$ with the two disks which constitute the intersection of $\operatorname{Bd}(N(D))$ with the inside of $M$. The resulting torus $M^{\prime}$ is incompressible to the inside, because if not, the inside of $M$ would be a cube-with-two-handles, contradicting the knottedness of $S$. Since $M^{\prime} \cap S=\varnothing, M^{\prime}$ is not boundary parallel. Let $D^{\prime}$ be a disk in the outside of $M^{\prime}$, with $D^{\prime} \cap M^{\prime}=\operatorname{Bd}\left(D^{\prime}\right)$. Put $D^{\prime}$ in general position with $A$ and assume $D^{\prime}$ has been moved by an isotopy until $A \cap D^{\prime}$ is minimal. As above, we may assume that $D^{\prime} \cap S=\varnothing$, so each component of the intersection of $D^{\prime}$ with the outside of $M$ is parallel to a subset of $M$, and we can thus remove any component of $A \cap D^{\prime}$. Hence $D^{\prime}$ is parallel to a disk in $M^{\prime}$, and $M^{\prime}$ is incompressible, contradicting the simplicity of our knot. It follows that $M$ is incompressible.

\section{BIBLIOGRAPHY}

1. W. R. Alford, Complements of minimal spanning surfaces of knots are not unique, Ann. of Math. (2) 91 (1970), 419-424. MR 40 \#6527.

2. W. R. Alford and C. B. Schaufele, Complements of minimal spanning surfaces of knots are not unique. II. Topology of Manifolds (Proc. Inst., Univ. of Georgia, Athens, Ga., 1969), Markham, Chicago, Ill., 1970, pp. 87-96. MR 44 \#5947.

3. R. J. Daigle, Complements of minimal surfaces, University of Georgia, Athen s, $\mathrm{Ga}$. (preprint).

4. - More on complements of minimal spanning surfaces, Rocky Mountain J. Math. 3 (1973), 473-482. 
5. R. H. Fox, A quick trip through knot theory, Topology of 3-Manifolds and Related Topics (Proc. Univ. of Georgia Inst. 1961), Prentice-Hall, Englewood Cliffs, N.J., 1962, pp. 120-167. MR 25 \#3522.

6. Herbert C. Lyon, Incompressible surfaces in knot spaces, Ph.D. Dissertation, University of Michigan, Ann Arbor, Mich., 1970.

7. - Incompressible surfaces in knot spaces, Trans. Amer. Math. Soc. 157 (1971), 53-62. MR 43 \#1169.

8. Horst Schubert, Knoten und Vollringe, Acta Math. 90 (1953), 131-286. MR 17, 291.

Unesco at The University of Jordan, Amman, The Hashemite Kingdom of JORDAN

Current address: Department of Mathematics, University of Michigan-Flint, Flint, Michigan 48503 